Acta Crystallographica Section A

Foundations of Crystallography

ISSN 0108-7673

Received 16 November 2012

Accepted 13 April 2013

\title{
Digital electron diffraction - seeing the whole picture
}

\author{
Richard Beanland, ${ }^{\mathrm{a} *}$ Paul J. Thomas, ${ }^{\mathrm{b}}$ David I. Woodward, ${ }^{\mathrm{a}}$ Pamela A. Thomas ${ }^{\mathrm{a}}$ and \\ Rudolf A. Roemer ${ }^{\text {a }}$
}

${ }^{\mathbf{a}}$ Department of Physics, University of Warwick, Coventry CV4 7AL, England, and ${ }^{\mathbf{b}}$ Gatan UK Ltd, 25 Nuffield Way, Abingdon, Oxon OX14 1RL, England. Correspondence e-mail:

r.beanland@warwick.ac.uk

\begin{abstract}
The advantages of convergent-beam electron diffraction for symmetry determination at the scale of a few $\mathrm{nm}$ are well known. In practice, the approach is often limited due to the restriction on the angular range of the electron beam imposed by the small Bragg angle for high-energy electron diffraction, i.e. a large convergence angle of the incident beam results in overlapping information in the diffraction pattern. Techniques have been generally available since the 1980s which overcome this restriction for individual diffracted beams, by making a compromise between illuminated area and beam convergence. Here a simple technique is described which overcomes all of these problems using computer control, giving electron diffraction data over a large angular range for many diffracted beams from the volume given by a focused electron beam (typically a few $\mathrm{nm}$ or less). The increase in the amount of information significantly improves the ease of interpretation and widens the applicability of the technique, particularly for thin materials or those with larger lattice parameters.
\end{abstract}

\section{Introduction}

The weak interaction of X-rays and neutrons with matter makes them ideal for structure solution of bulk materials (of size $\gtrsim 10 \mu \mathrm{m}$ ) since single scattering events dominate, but results in a low scattering intensity from small volumes. Conversely, the strong interaction of electrons with matter allows analysis of nanoscale volumes, but complicates their use due to the dominance of multiple (dynamical) scattering events. The specimen must be very thin (typically $<200 \mathrm{~nm}$ ) to allow transmission of the electron beam, usually in a transmission electron microscope (TEM). Dynamical scattering causes the diffracted intensity for any given reflection $h k l$ from a crystalline material to vary enormously as a function of the incident-beam orientation, even when the Bragg condition is satisfied exactly. It also produces significant intensity in reflections that are completely absent in singly scattered diffraction (i.e. kinematically 'forbidden' reflections). This broadly prevents the simple use of electron diffraction patterns for structure solution. Nevertheless, the symmetry of an electron diffraction pattern is still determined by the symmetry of the crystal from which it is produced, and dynamical scattering has some distinct advantages, such as information describing the phase of the diffracted electrons (Spence, 1993; Tanaka \& Tsuda, 2011), sensitivity to chirality (Johnson, 2007) and the breaking of Friedel's law (Friedel, 1913; Steeds \& Vincent, 1983). Friedel's law generally holds for X-ray and neutron scattering (with the exception of anomalous absorption effects) and renders diffraction data insensitive to the presence of a centre of symmetry in a crystal. These factors, together with a greater sensitivity to valence electron densities (Spence, 1993; Zuo, 2004), mean that electron diffraction data are in principle richer and more sensitive than those from other techniques.

The description of electron diffraction using dynamical scattering theory is well established and the difficulties do not lie in a lack of a well understood theory or low signal strength; rather, the main challenge is often to extract a sufficient quantity of data to allow dynamical theory to be applied with confidence. At the heart of the problem is the fact that, because of the very small wavelength of high-energy electrons, Bragg angles, $\theta_{h k l}$, for diffracted electron beams are small (typically less than $1^{\circ}$ ), while diffraction can occur for most strong reflections at large deviations ( $>2 \theta_{h k l}$ or more) from the Bragg condition. This inevitably leads to overlapping diffracted beams unless the half-convergence angle, $\alpha$, of incident illumination is restricted to be less than the smallest Bragg angle in any given convergent-beam electron diffraction (CBED) pattern, a fact which has been appreciated since the very beginning of electron diffraction (Kossel \& Möllenstedt, 1939). This 'overlap problem' severely restricts the angular range of data that can be obtained, particularly from materials with relatively large lattice parameters. In general, the thinner the specimen and the larger the lattice parameter, the fewer features become visible in CBED patterns, giving only a set of almost blank discs. In combination with some (almost inevi- 

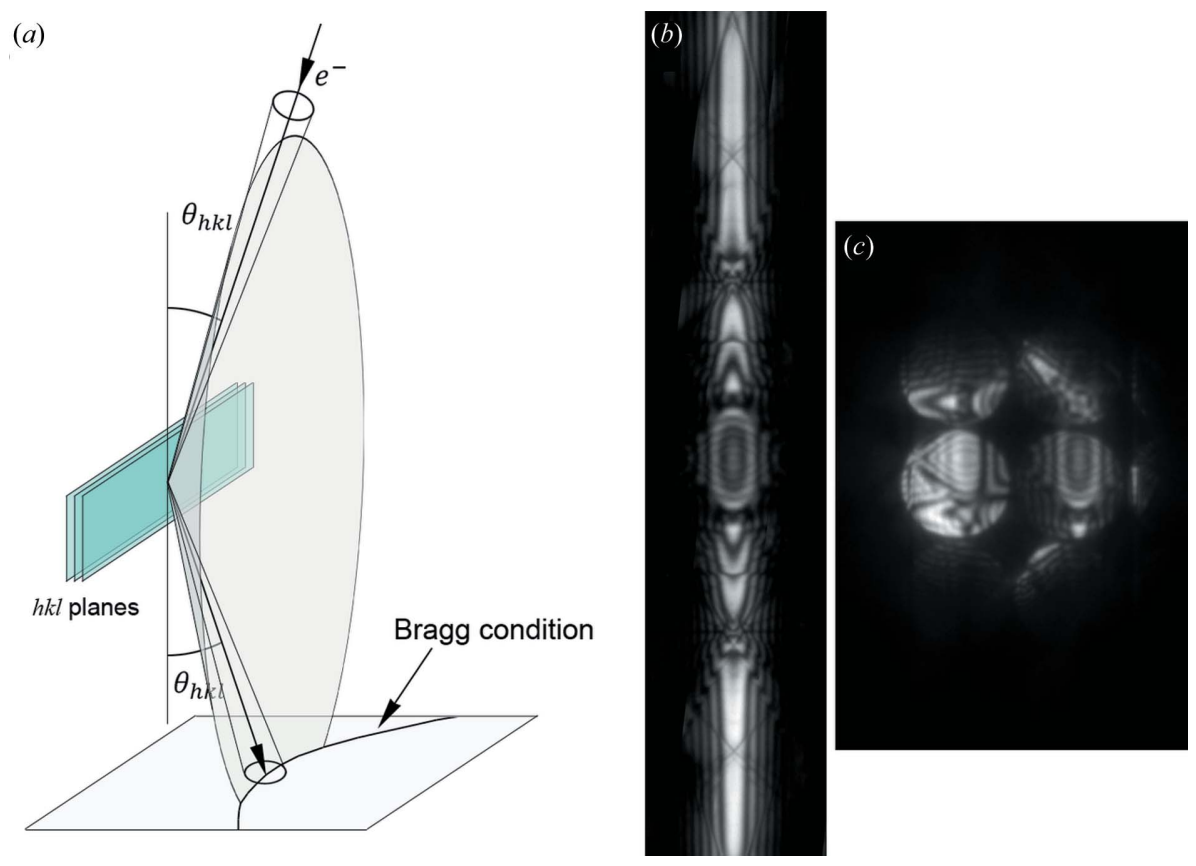

\section{Figure 1}

(a) The geometry of large-angle convergent-beam electron diffraction (LACBED) for one diffracted beam, ignoring all other diffracted beams. The Bragg condition is satisfied when the incident and exit beams make an angle $\theta_{h k l}$ to the diffracted planes, defining a cone. This gives a parabola on the diffraction pattern which, because of the very small Bragg angle, appears as a straight line in an LACBED pattern $(b)$, taken from a [100] silicon crystal. $(c)$ The corresponding CBED pattern, with a small part of the LACBED pattern visible in the $2 \overline{2} 0$ disc.

below the specimen, to produce a large number of low-resolution LACBED patterns, again captured in a single exposure on camera. Thus, to date, almost all $^{\mathbf{1}}$ (Terauchi \& Tanaka, 1985) electron diffraction techniques sample only a very limited part of the full dynamical diffraction data set.

Here, we describe a method which eliminates the fundamental problem of beam overlap using computer control, providing large diffraction data sets with many diffracted beams which contain detailed information from a region as small as the electron beam focused on the specimen, which can be a few $\mathrm{nm}$ in size or less.

\section{Methods}

If the problem of beam overlap can be neglected, a dark-field LACBED pattern takes the form of a bright line of diffracted intensity, corresponding to the angle at which the Bragg condition is satisfied (Fig. 1). A small portion of this pattern is visible in one disc of a CBED pattern (Fig.

table) bending of a thin specimen, this means that the exact orientation of the crystal is difficult to determine and interpretation becomes more and more demanding.

The overlap problem was partially solved by Tanaka et al. (1980) using a highly convergent beam and a displacement of the specimen from the image plane of the objective lens in a TEM, blocking all diffracted beams apart from the one of interest by placing an aperture in a conjugate image plane. A slightly different solution was developed by Eades (1980) using a parallel-beam rocking both above and below the specimen in STEM (scanning transmission electron microscopy) mode. Both of these approaches give access to a more complete diffraction data set for one diffracted beam. However, such large-angle convergent-beam (LACBED) patterns obtained using the Tanaka method can only be obtained from large, parallel-sided, flat crystals unless an unusually small aperture is used (Tanaka \& Tsuda, 2011) (any bending of the crystal leads to distortions in the pattern) and the scanning method is difficult to implement. Furthermore, both of these techniques only allow access to one diffracted beam at a time; obtaining several LACBED images is both time consuming and requires significant effort and skill on the part of the operator. Recently, Koch (2011) used computer control of the microscope to rock a parallel beam in a similar way to Eades, together with partial compensation of the tilt

\footnotetext{
${ }^{1}$ Access to diffraction patterns which contain both a large angular range and multiple diffracted beams was demonstrated by Terauchi \& Tanaka (1985) using a TEM in an unusual configuration. This feat does not appear to have been repeated since.
}

1c). It is immediately apparent that, by collecting a large number of individual CBED patterns with different incidentbeam tilts, the LACBED pattern can be reconstructed by combining the relevant parts of each individual CBED pattern as shown in Fig. 2. We implemented this approach using a JEOL 2100 TEM operating at $200 \mathrm{kV}$ with standard computer control of the electron-optic lenses and a digital camera. Standard conditions for CBED were used, i.e. the electron beam was focused to a small probe (typically $\sim 15 \mathrm{~nm}$ FWHM) on a thin specimen, with the illumination convergence angle adjusted such that there was no significant overlap of the discs in the diffraction pattern. The tilt of the incident beam was controlled via a computer script to scan over a large angular range (typically up to 0.1 radians, or $\sim 5.7^{\circ}$ ), corresponding to approximately $50 \mathrm{~nm}^{-1}$, and a diffraction pattern was collected at each different incidentbeam tilt using the CCD camera. The beam-tilt step was adjusted to give $\sim 30 \%$ overlap between consecutive patterns. The exposure time for an individual CBED pattern was typically $40 \mathrm{~ms}$, although camera processing overhead increased the time between individual frames to approximately $80 \mathrm{~ms}$ (i.e. 1000 patterns in $80 \mathrm{~s}$ - sufficiently fast to avoid problems with specimen drift or contamination). In this microscope the upper limit of the tilt range which can be obtained without computer control is due to the spherical aberration of the pre-field objective lens, causing shifts and changes in beam shape for incident-beam tilts much more than 60-100 mrad from the optic axis, depending upon the excitation of the final condenser mini-lens. Careful beam-tilt 

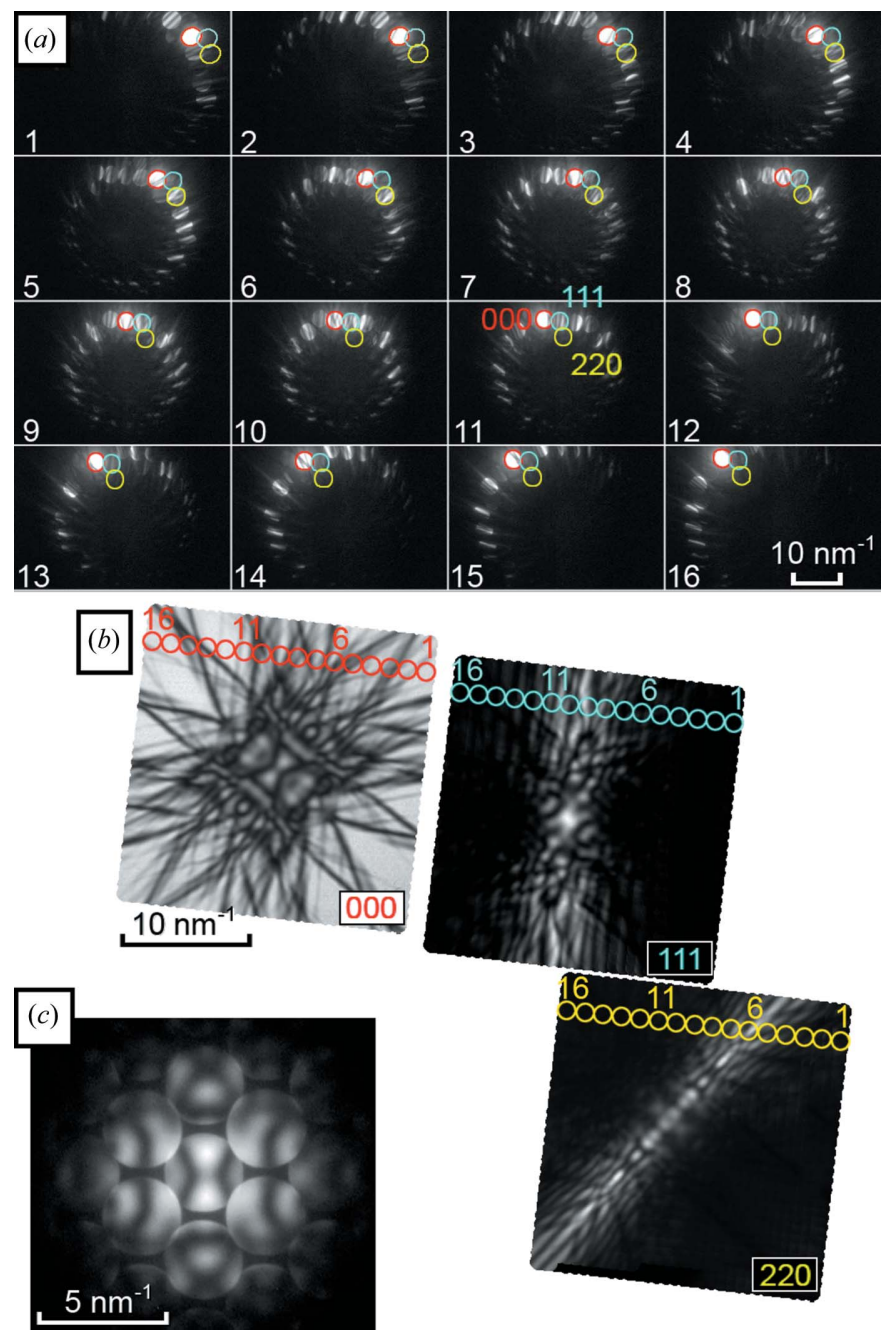

Figure 2

(a) Sixteen CBED patterns from [110] silicon with varying beam tilts. The 000 (red), 111 (blue) and 220 (yellow) beams are highlighted in each. (b) Digital reconstruction of LACBED patterns from many individual CBED patterns, highlighting the components from the patterns in $(a)$. (c) The on-axis CBED pattern.

compensation using a look-up table (see the supplementary material $^{2}$ ) was employed to ensure that beam shift during data acquisition was less than $20 \%$ of the FWHM of the electron beam $(\sim 3 \mathrm{~nm})$ for the maximum beam tilt used in any given data set. The data from each different diffracted beam were then recombined into a single image using a second computer script, giving a montage of digital LACBED (D-LACBED) patterns. For this angular range, useful D-LACBED patterns can be extracted for typically 50-60 different reflections from a single data set.

\section{Results}

We begin with data from 'standard' materials GaAs, Si and $\alpha-\mathrm{Al}_{2} \mathrm{O}_{3}$, which have often been used for conventional CBED

\footnotetext{
${ }^{2}$ Supplementary material for this paper is available from the IUCr electronic archives (Reference: TD5013). Services for accessing these data are described at the back of the journal.
}

investigations. All specimens were large single crystals, ion milled to electron transparency using conventional specimen preparation methods. Fig. 3 shows the central 17 patterns obtained from [1]0] GaAs. The D-LACBED patterns are arranged such that they have the same relative positions as in the conventional electron diffraction patterns, although note that each D-LACBED pattern covers an angular range similar to the whole of the conventional patterns shown for comparison to the left. The relationship between the symmetries of an electron diffraction pattern and those of the crystal was determined by Buxton et al. (1976) and is based upon the premise that all of the information visible in Fig. 3 is available. Normally, when performing such a symmetry determination using CBED, the skill and time needed carefully to choose the specimen thickness, as well as manual tilting of the incident beam and/or specimen to allow different parts of each darkfield pattern to be observed, are considerable. The ease of a single click for data collection, and the significant improvement in symmetry identification that results from access to a larger part of the complete dynamical diffraction data set, is readily apparent.

Since no higher-order Laue zone (HOLZ) lines are visible in Fig. 3, the pattern symmetries (both bright-field and darkfield) correspond to a projection of the crystal (Buxton et al., 1976). The directly transmitted beam may in general have higher symmetry than the pattern as a whole, and this is the case here with the $\mathbf{g}=000$ D-LACBED pattern having symmetry $2 \mathrm{~mm}$. Each individual dark-field pattern, corresponding to a different diffracted beam, can have symmetry up to $2 \mathrm{~mm}$ in itself; in Fig. 2 it can be seen that this is only the case for those patterns crossing the vertical (110) mirror plane, i.e. $\mathbf{g}$ =002-type patterns (note that careful inspection of the 220type D-LACBED patterns reveals the lack of any mirror). All other patterns, i.e. $\mathbf{g}=111,222,220$ and 113 type, have twofold symmetry about their centre. This symmetry operation, denoted $1_{R}$ (Buxton et al., 1976), can indicate the presence of a mirror plane perpendicular to the electron beam; however, it is also present in all zero-order Laue zone (ZOLZ) reflections (as is the case here) since the projected potential of the crystal is independent of the sense of the electron-beam direction. Therefore in this case the presence or absence of a mirror perpendicular to the electron beam cannot be determined. There is no horizontal (001) mirror present, indicating the polarity of the crystal, and the lack of equivalence in $\pm \mathbf{g}$ pairs, indicating the lack of a centre of symmetry, is obvious. As a whole, the pattern has symmetry $m$ and projection diffraction group $m 1_{R}$, as expected for a crystal with space group $F \overline{4} 3 m$ and point group $\overline{4} 3 m$.

The multiple scattering processes which are inherent to electron diffraction, in combination with a limited sampling of the dynamical diffraction data set, often give rise to the impression that electron diffraction is unreliable or limited in application in comparison with X-ray crystallography. However when the structure is well known, as in the case of $\mathrm{GaAs}$, it is straightforward to reproduce the experimental data using standard simulation software (Stadelmann, 1987), as illustrated by Fig. 4. 


\section{research papers}
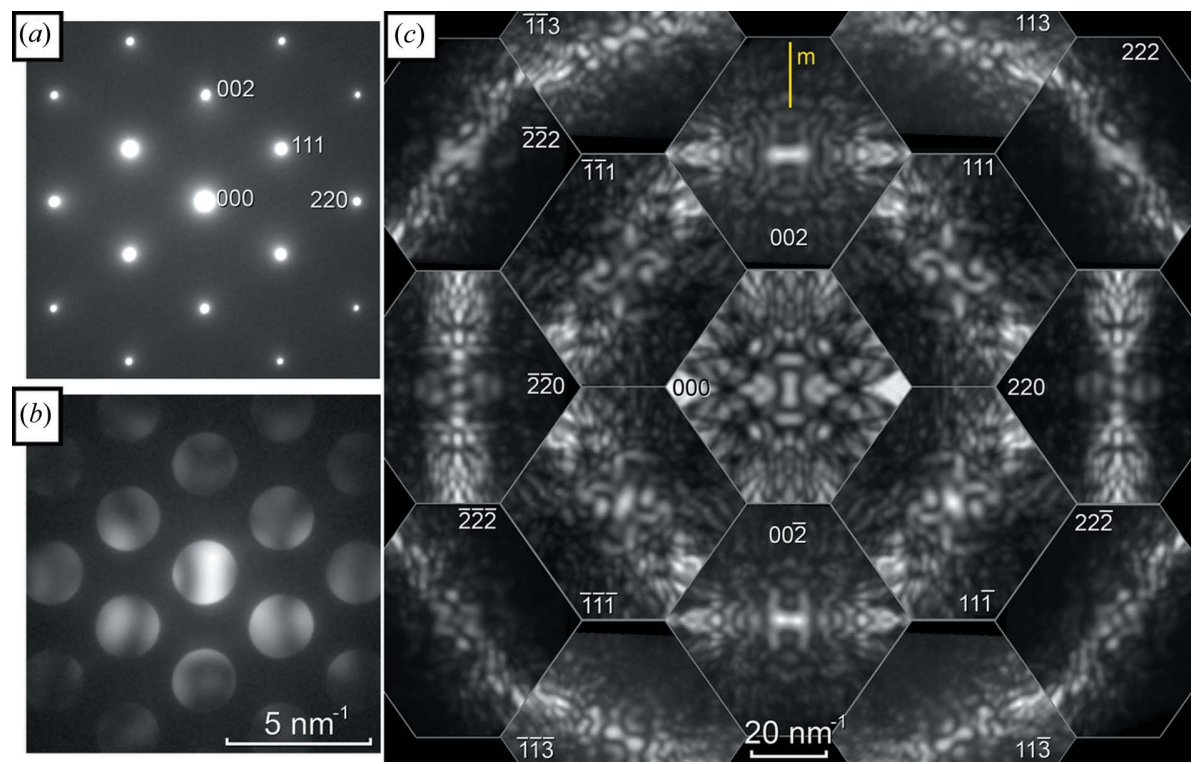

Figure 3

(a) Selected-area electron diffraction (SAED), (b) CBED and (c) a montage of 17 D-LACBED patterns taken from [1 $1 \overline{1} 0] \mathrm{GaAs}$; the patterns are arranged in positions corresponding to the CBED pattern. The diffraction vector $\mathbf{g}$ is indicated for each pattern and the (110) mirror plane is indicated by the letter $m$; no horizontal mirror is present. The whole-pattern symmetry is $m$ and the projection diffraction group is $m 1_{R}$.

A similar D-LACBED montage taken from [110]0] silicon, with space group $F d \overline{3} m$ and point group $m \overline{3} m$, is shown in Fig. 5. The equivalence between the two face-centred cubic sublattices in the diamond structure doubles the number of symmetry elements in the crystal space group (including the addition of a centre of symmetry, giving an equivalence between $\pm \mathbf{g}$ pairs) but also leads to kinematically forbidden reflections with indices $\mathbf{g}=002,222,442, \ldots$ (Morniroli \& Ji,

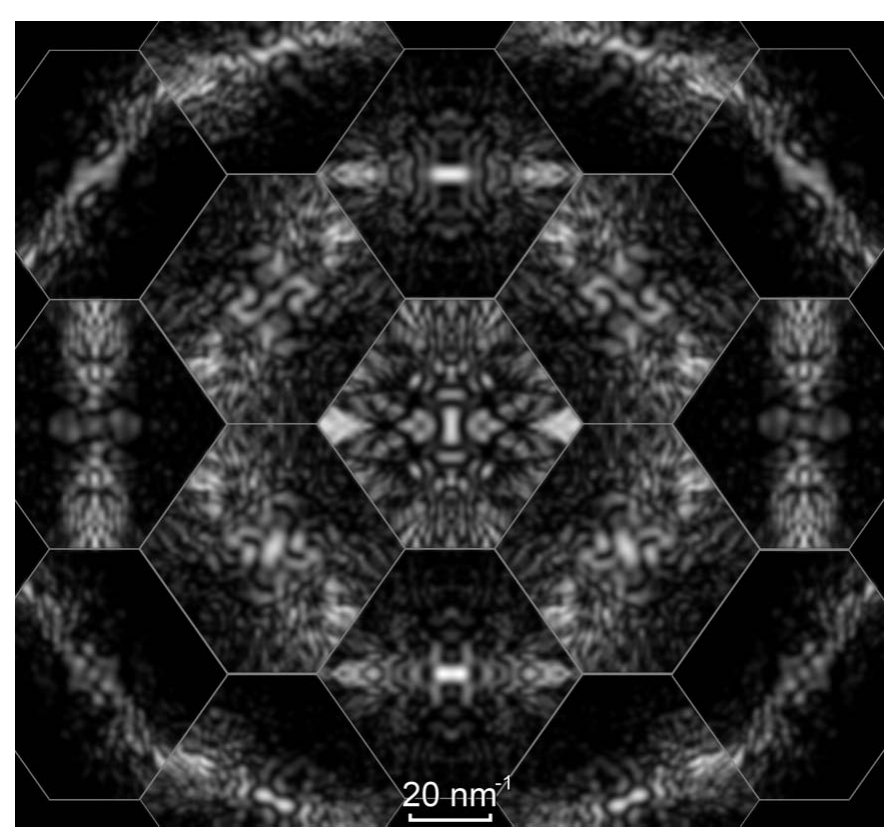

Figure 4

Montage of simulated LACBED patterns corresponding to the experimental data of Fig. 3 at a specimen thickness of $85 \mathrm{~nm}$.
2009). In conventional electron diffraction these forbidden reflections often appear just as strongly as the 'allowed' reflections, as can be seen in Fig. 5(a). Nevertheless, the 002 reflection should drop to zero intensity at angles sufficiently far away from a zone axis [as employed in precession electron diffraction (Vincent \& Midgley, 1994)] where multiple scattering pathways do not exist. This is indeed the case and is clearly visible in D-LACBED data, as shown in Fig. 5(c).

Figs. 3-5 do not show reflections from HOLZs and only their projection diffraction group can be given. This can lead to some ambiguity in space-group determination, since there are usually more point groups which are consistent with a given projection diffraction symmetry than those consistent with a pattern containing three-dimensional information. However, D-LACBED can access the same three-dimensional information as conventional CBED, as demonstrated in Fig. 6. This shows a trio of diffraction patterns from [5051] $\alpha-\mathrm{Al}_{2} \mathrm{O}_{3}$. HOLZ lines are clearly visible in the central disc of the CBED pattern, although all reflections which do not lie on the $\overline{1} 2 \overline{1} 0$ systematic row are too weak to be seen (Fig. 6b). There are kinematically forbidden reflections caused by the $c$-glide plane in the $R 3 c$ space group, indicated by circles overlaid on the selected-area electron diffraction (SAED) pattern (Fig. 6a). Since the digital diffraction pattern is a combination of many individual CBED patterns, the HOLZ lines are also present in the D-LACBED pattern (Fig. $6 c$ ). Ignoring HOLZ lines, the projection symmetry of the bright-field D-LACBED pattern is $2 \mathrm{~mm}$ and the projection diffraction group is $2 m m 1_{R}$, whereas with the HOLZ lines these are reduced to a bright-field symmetry of $m$ and diffraction group $2_{R} m m_{R}$. As before, the symmetry of the D-LACBED pattern is easier to distinguish and more informative than either the CBED or SAED patterns; in particular the kinematically forbidden nature of the $\overline{1} 10 \overline{5}$ reflections is immediately evident. In general, we find that kinematically forbidden reflections are readily identified in D-LACBED data sets, even when the crystal is relatively thick and multiple scattering dominates.

While it is useful to see the large improvement in data available when examining $\mathrm{GaAs}, \mathrm{Si}$ and $\alpha-\mathrm{Al}_{2} \mathrm{O}_{3}$, the real utility of the technique lies in its application to nanostructured materials which are difficult to tackle using X-ray diffraction, or even conventional electron diffraction. We therefore consider a material which has unknown symmetry: $\mathrm{NaBi}$ $\mathrm{CaTeO}_{6}$, an $\left(A^{3+} A^{1+}\right) B^{2+} \mathrm{TeO}_{6}$ material that we take here to be an example of a typical perovskite oxide. The prototype perovskite structure is cubic, with symmetry $\operatorname{Pm} \overline{3} m$ and lattice parameter typically around $0.4 \mathrm{~nm} ; \mathrm{NaBiCaTeO}_{6}$ might be 


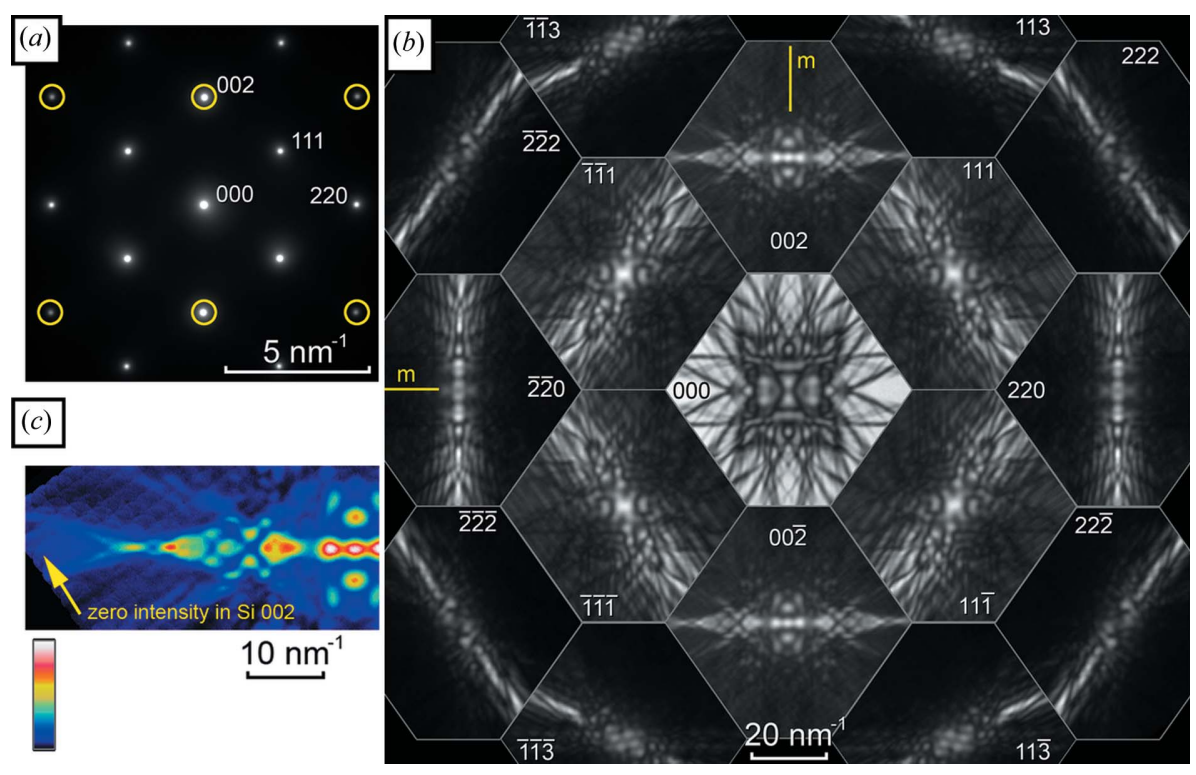

Figure 5

(a) SAED and $(b)$ a montage of 17 D-LACBED patterns taken from [11̄0] silicon. Highlighted reflections in the SAED pattern are forbidden and would have zero intensity without multiple scattering. Part $(c)$ shows the 002 D-LACBED pattern at a large angle from the zone axis and it is clear that the intensity does indeed drop to zero. The whole-pattern symmetry is $2 \mathrm{~mm}$ and the projection diffraction group is $2 m m 1_{R}$.
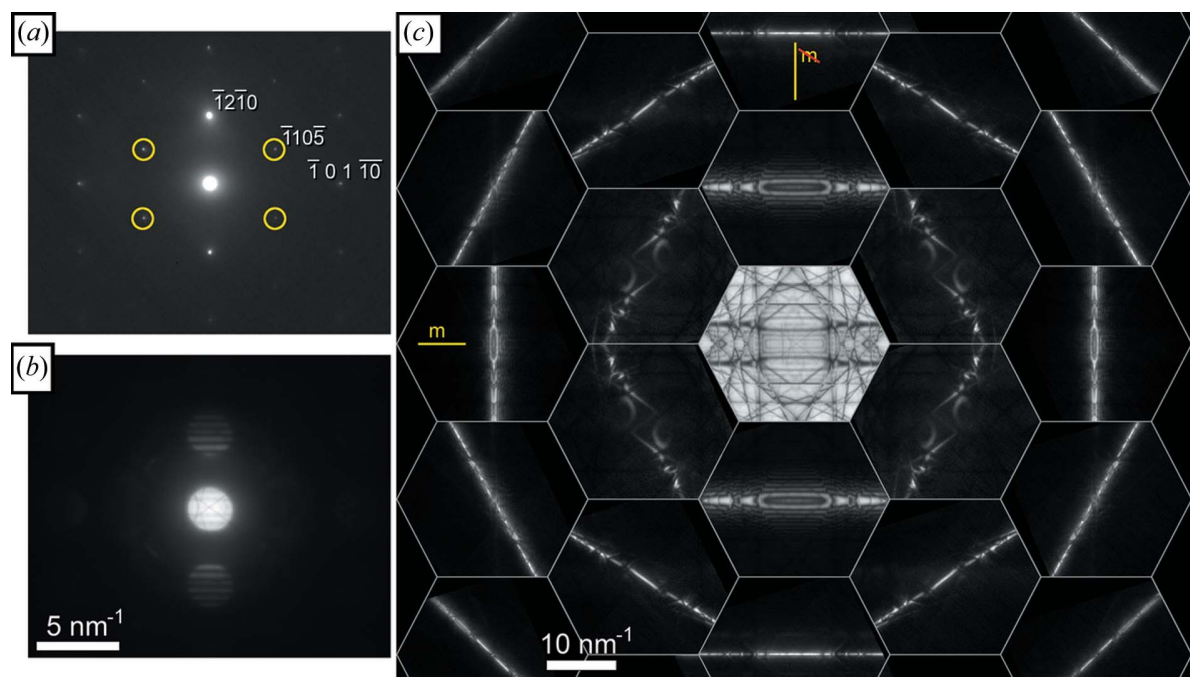

Figure 6

(a) SAED, $(b)$ CBED and $(c)$ a montage of 23 D-LACBED patterns taken from [5051] $\alpha-\mathrm{Al}_{2} \mathrm{O}_{3}$. Highlighted reflections in the SAED pattern are kinematically forbidden. The presence of HOLZ lines breaks the vertical mirror symmetry present in the ZOLZ. The whole-pattern symmetry is $m$ and the diffraction group is $2_{R} m m_{R}$.

expected to exhibit $A$ - and/or $B$-cation ordering, and/or displacements from nominal positions in the unit cell, and/or tilting of the oxygen octahedra (Glazer, 1972) or any combination of these effects (Howard \& Stokes, 2004; Howard \& Zhang, 2004; Kishida et al., 2009). In any case, we expect the space group to be some subgroup of $\operatorname{Pm} \overline{3} \mathrm{~m}$. Tellurium-containing compounds can exhibit ferroelectric or antiferroelectric behaviour (Venevtsev et al., 1974; Politova \& Venevtsev, 1975); in terms of functional properties, ferroelectric behaviour is preferable since this leads to piezoelectric, pyroelectric and other useful applica- tions. Since these ferroic properties only exist in materials without a centre of symmetry, determination of the crystal point and space group has direct relevance to technological utility, and electron diffraction has a distinct advantage here. A priori determination of crystal space group from dynamical electron diffraction patterns has been described by Goodman (1975), Steeds \& Vincent (1983), Tanaka \& Tsuda (2011) and, more recently, Morniroli et al. (2012) and Jacob et al. (2012), all of whom rely on the original classification of dynamic diffraction symmetries of Buxton et al. (1976). As the structure is unknown, we will use a pseudocubic notation (i.e. treat the crystal as if it were a prototype perovskite, for indexing purposes only). Data were collected from defect-free domains in a polycrystalline ceramic (grain size typically $<1 \mu \mathrm{m}$ ), prepared for transmission electron microscopy using standard techniques, and similar probe sizes were used as in the $\mathrm{Si}$ and GaAs examples. However, significantly smaller convergence angles were required due to the larger lattice parameter encountered.

Fig. 7 shows electron diffraction patterns from a $\langle 100\rangle_{P C}$ axis, denoted here $[100]_{P C}$. Half-order $h k l / 2$ ('superstructure') spots are visible in the SAED pattern (Fig. $7 a$ ), where $h, k, l$ are integers, often described as half 'even-even-odd' or $\frac{1}{2}$ eeo spots (Woodward \& Reaney, 2005; Reaney et al., 1994), indicating a doubling of periodicity along $[001]_{P C}$ but not $[010]_{P C}$. In the CBED discs of Fig. $7(b)$, some bright and dark regions are present with no apparent symmetry, but it is not clear if this is simply because the crystal is not aligned exactly with the incident electron beam. The information available is rather limited. Conversely, much more information is easily extracted from the montage of Fig. 7(c), which clearly shows a lack of any mirror symmetry, twofold symmetry in all individual D-LACBED patterns (due to projection) and projection diffraction group $21_{R}$. It is clear that (sub)unit-cell distortions and/or cation ordering have broken all $\{110\}_{P C}$ and $\{100\}_{P C}$ mirrors that could be present in this pattern. The $21_{R}$ projection symmetry of Fig. 7 is consistent with the presence of a twofold axis along the beam direction, or a centre of symmetry in the crystal, or both; the lack of any three- 


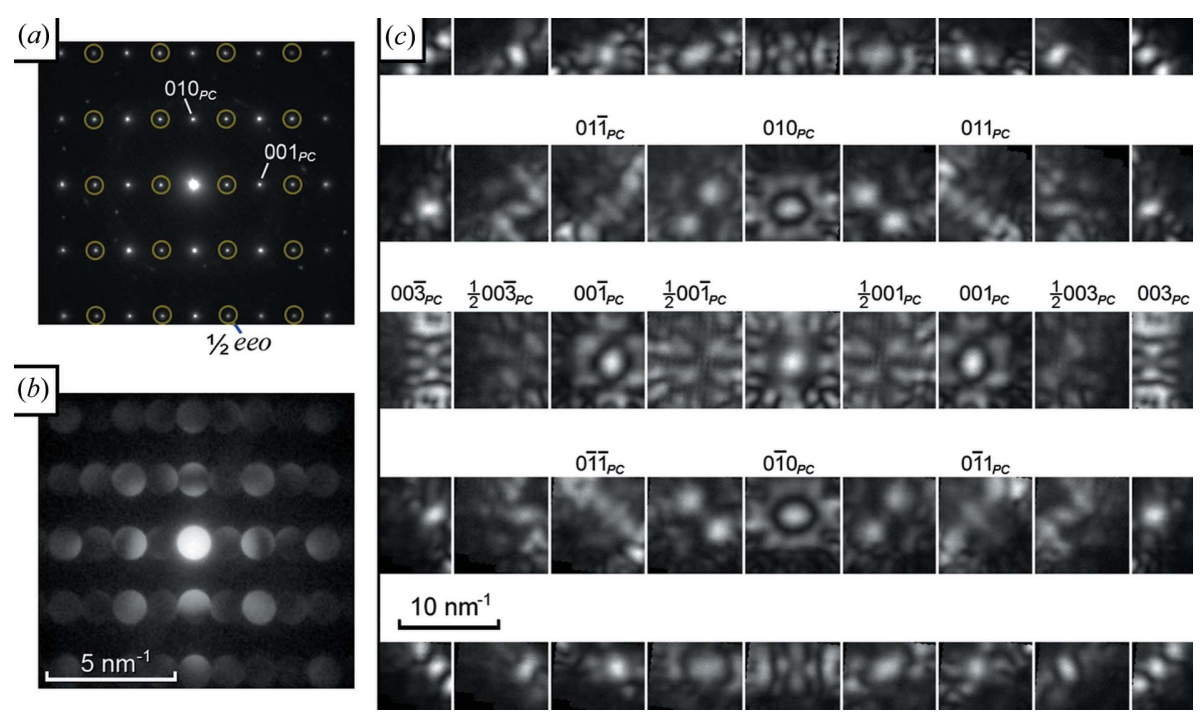

Figure 7

(a) SAED, (b) CBED and (c) a montage of 45 D-LACBED patterns taken from $[100]_{P C}$ $\mathrm{NaBiCaTeO}_{6}$. The whole-pattern symmetry is 2 and the projection diffraction group is $21_{R}$.
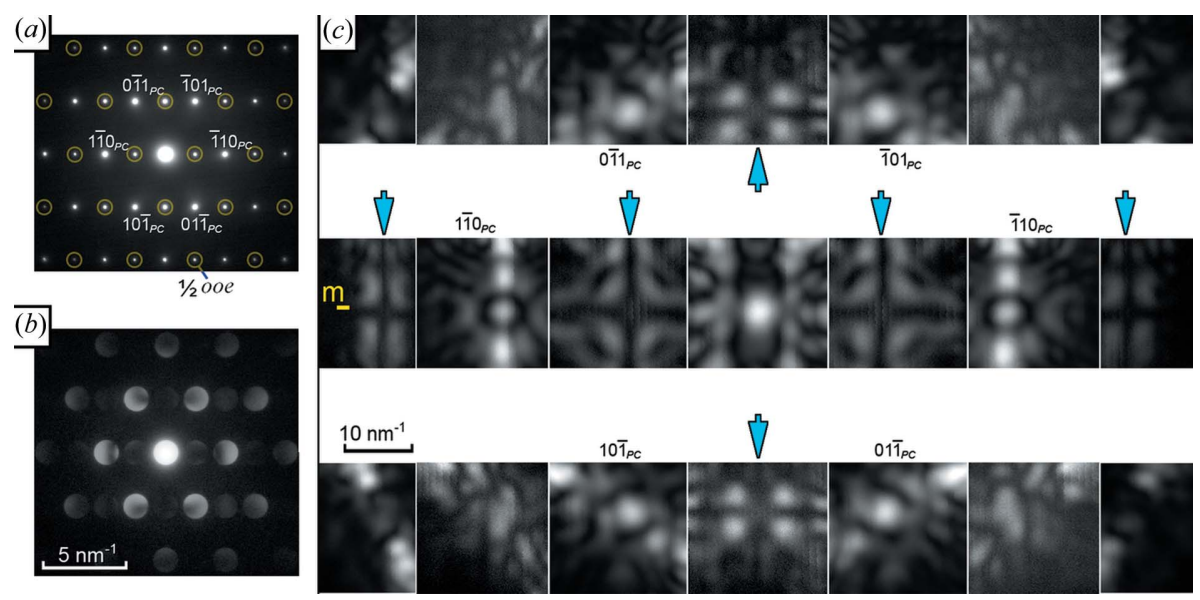

Figure 8

(a) SAED, (b) CBED and (c) montage of 21 D-LACBED patterns taken from $[111]_{P C}$ $\mathrm{NaBiCaTeO}_{6}$. Arrows mark Gjønnes-Moodie lines, indicating the presence of a $2_{1}$ axis along $[\overline{1} 10]_{P C}$ and a $\left(\overline{1} 10_{P C}\right.$ mirror-glide plane. The whole-pattern symmetry is $2 \mathrm{~mm}$ and the projection diffraction group is $2 m m 1_{R}$.

dimensional information in the form of HOLZ lines prevents them from being distinguished in this case.

Fig. 8 shows a similar trio of diffraction patterns from a $[111]_{P C}$ axis, taken from a different crystal. Here, $\frac{1}{2}$ ooe superstructure spots are visible in the SAED pattern; again there is little detail in the CBED discs and the superstructure discs are weak but visible. Once more, an enormous amount of information is visible in the D-LACBED pattern. Strikingly obvious black crosses are visible in alternate patterns along the horizontal systematic row, i.e. $\frac{1}{2} 0 \overline{1} 1_{P C^{-}}$and $\frac{1}{2} 0 \overline{3} 3_{P C^{-}}$type patterns. They are also present along the vertical $\frac{1}{2} \overline{11} 2_{P C}$ systematic row. These dark crosses are dynamical extinction effects (Gjønnes \& Moodie, 1965; Tanaka et al., 1987), often known as Gjønnes-Moodie lines, and are present when the incident electron beam is parallel to a glide plane or perpendicular to $2_{1}, 4_{1}, 4_{3}, 6_{1}, 6_{3}$ and $6_{5}$ screw axes. Whilst in principle these could be observed in CBED patterns, this is difficult when the crystal is thin and the beam-convergence angle is small; not enough of the diffraction data set is visible. It is interesting to compare these patterns with the $\frac{1}{2} 100_{P C}$ D-LACBED patterns in Fig. 7, which also contain a dark cross - however, the $\frac{1}{2} 300_{P C}$ D-LACBED patterns have no black cross and we therefore do not consider them to indicate the presence of a glide plane or screw axis.

Fortuitously in this case, the D-LACBED data from just these two zone axes - and the knowledge that the crystal is a perovskite - are sufficient to determine the point group of the crystal. As it is a perovskite, the actual space group of the ordered and/or distorted crystal must be a subgroup of the prototype perovskite space group $P m \overline{3} m$ (Howard \& Stokes, 1998). The allowable point groups, subgroups of $m \overline{3} m$, are given in Fig. 9. The GjønnesMoodie crosses in the $[111]_{P C}$ pattern indicate the presence of perpendicular glide planes or screw axes; examination of the stereographic representation of the point group $m \overline{3} m$ shows the lack of fourfold or sixfold axes perpendicular to $[111]_{P C}$ in the prototype point group, giving only one possibility - i.e. a point group of (at least) $2 / m$, with a $22_{1}$ screw axis along $[\overline{1} 10]_{P C}$ and perpendicular glide plane. Nevertheless, this single measurement of projected symmetry along one direction is not sufficient by itself, since there are five subgroups of $m \overline{3} m$ that contain $2 / m$ (Fig. 9). These point groups of higher order would give the same, or higher, symmetry in the [111] pattern and in general the projected symmetry at other zone axes must be considered in order to arrive at a unique solution. The point group $m \overline{\mathbf{3}}$ can be eliminated immediately since this would require threefold symmetry at all [111 $]_{P C}$ zone axes, and the group $4 / m$ which contains $2 / m$, with the fourfold axis along $[\overline{1} 10]_{P C}$, is not a subgroup of $m \overline{3} m$ and can also be eliminated. The point group $4 / \mathrm{mmm}$ can only be reached through the intermediate $\mathrm{mmm}$, and so we consider the three cases $2 / m, m m m$ and $\overline{3} m$. The whole-pattern symmetries for ZOLZ patterns are straightforward to determine by inspection of the stereographic representations of the point groups in Fig. 9 and are given in Table 1. The point groups $m m m$ and $\overline{3} m$ would give whole-pattern symmetry of $2 \mathrm{~mm}$ in all $\langle 100\rangle_{P C}$ patterns; the observed $\langle 100\rangle_{P C}$ whole-pattern symmetry of 2 is thus only consistent with the point group $2 / m$ and observation along a $[100]_{P C}$ or $[010]_{P C}$ axis. We have chosen to index 
according to a $[100]_{P C}$ zone axis here. This analysis also indicates that there is no twofold axis along $[100]_{P C}$, the $21_{R}$ D-LACBED pattern symmetry of Fig. 7 is due to the centre of symmetry in the point group $2 / m$.

A third pattern is required to determine the translation vector of the glide plane, since dark Gjønnes-Moodie crosses are expected in the ZOLZ if there is any component of the glide vector perpendicular to the electron beam. We thus tilted the second crystal from the $[111]_{P C}$ orientation about the $2_{1}$ axis to the $[110]_{P C}$ zone axis (Fig. 10). The Gjønnes-Moodie
Table 1

Possible whole-pattern symmetries for projection D-LACBED patterns at the $[111]_{P C},[110]_{P C}$ and $\langle 100\rangle_{P C}$ zone axes (Figs. 7, 8 and 10) for the three possible crystal point groups that are consistent with $2 / m$ symmetry in a $[111]_{P C}$ diffraction pattern.

\begin{tabular}{llll}
\hline & \multicolumn{2}{l}{ Crystal point group } & \\
\cline { 2 - 4 } Zone axis & $2 / m$ & $m m m$ & $\overline{3} m$ \\
\hline$[111]_{P C}$ & $2 m m$ & $2 m m$ & $2 m m$ \\
{$[110]_{P C}$} & $2 m m$ & $2 m m$ & $2 m m$ \\
$\langle 100\rangle_{P C}$ & $2 m m$ or 2 & $2 m m$ & $2 m m$ \\
\hline
\end{tabular}
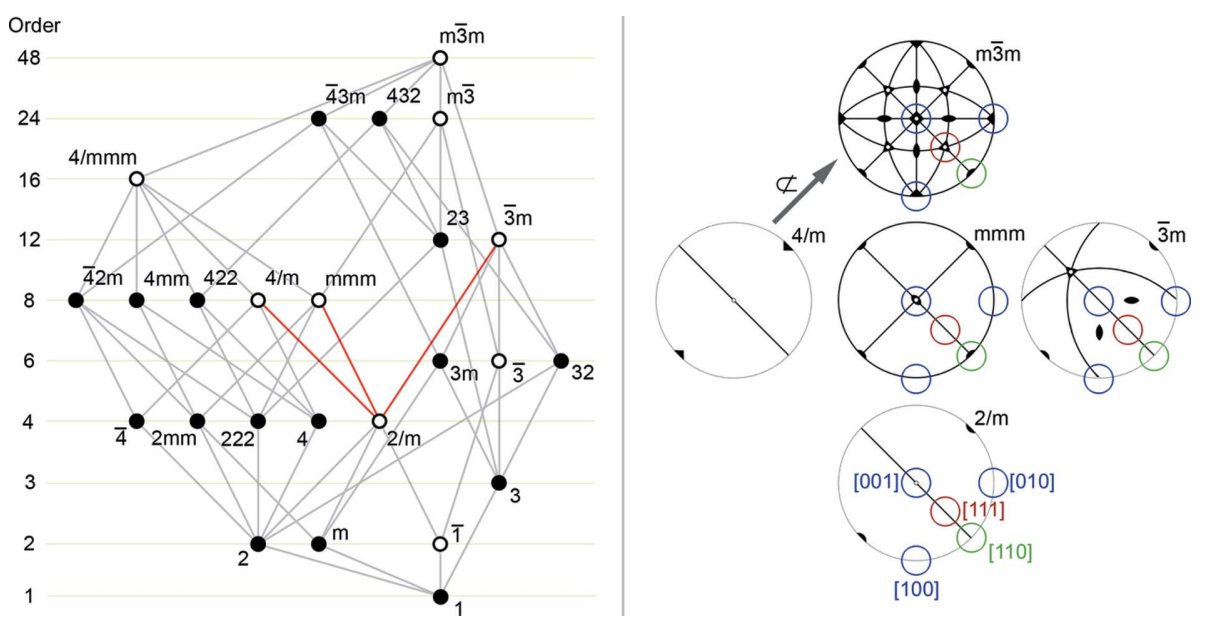

Figure 9

Left: point-group-subgroup relations for $m \overline{3} m$, with the relationship between the experimentally determined point group of $2 / m$ and groups of higher order highlighted. Right: stereographic projections of the point groups with symmetry elements and the three zone axes examined in this study.
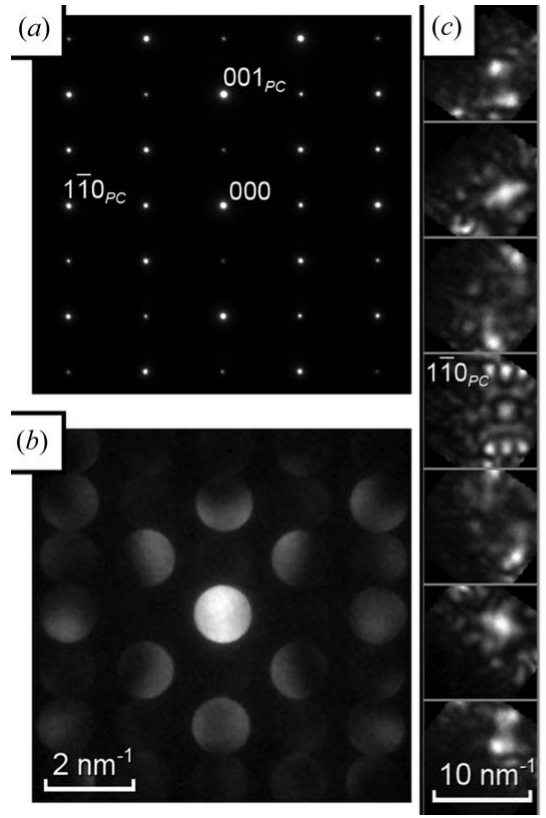
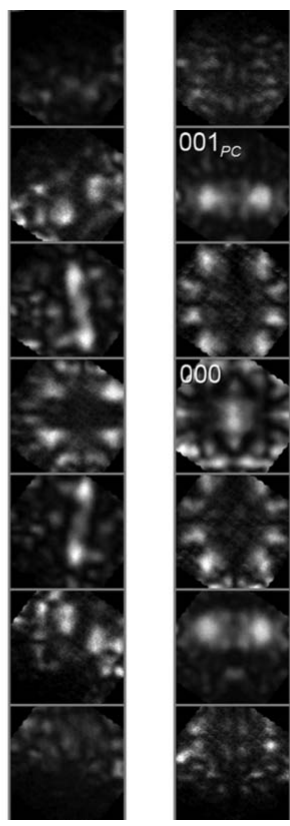

crosses remain (as expected) along the 21 axis $[\overline{1} 10]_{P C}$ and are also present along the perpendicular direction $[001]_{P C}$; the glide translation thus cannot be parallel to the $[110]_{P C}$ zone axis and can therefore only be parallel to $[001]_{P C}$. Finally, we note that in SAED patterns superstructure spots indicate a doubling of the $P$ lattice along two of the three pseudo-cubic axes. This fixes the space group as \#14, $P 2_{1} / c$, with the unique $b$ axis parallel to $[\overline{1} 10]_{P C}$, the $b$ axis parallel to $[110]_{P C}$ and the $c$ axis parallel to $[001]_{P C}$. The relative ease of determining the space group in this example is a direct result of the greater level of detail available in D-LACBED patterns in comparison with other diffraction techniques.

\section{Discussion}

We have demonstrated that computer control of beam tilt and image capture in a TEM can be used to overcome the problem of overlapping diffracted beams, quickly providing very rich diffraction data sets which can be used for easy determination of crystal symmetry on a nanometre scale. This approach stems from an appreciation of the fact that an image gathered from a CCD camera is a numerical data set which is easily combined with other data sets. The greatest experimental difficulty is to gather a suitable amount of data in a reasonable time, since specimen drift and contamination would render the data set meaningless. This is achieved using low-level programming to optimize the capture rate of CBED patterns. We typically achieve capture rates $>10$ patterns per second, most of the D-LACBED patterns shown here being a combination of up to a thousand individual (a) SAED, (b) CBED and $(c)$ a montage of 35 D-LACBED patterns taken from $[110]_{P C}$ $\mathrm{NaBiCaTeO}_{6}$. Gjønnes-Moodie dark crosses again mark the presence of a $2_{1}$ axis along $\left[\overline{1}_{10}\right]_{P C}$ and a $(\overline{1} 10)_{P C}$ mirror-glide plane. The whole-pattern symmetry is $2 \mathrm{~mm}$ and the projection diffraction group is $2 m m 1_{R}$. 
CBED patterns, acquired in less than $120 \mathrm{~s}$. It is clear that optimization of image capture and microscope control could easily improve on this, potentially reducing data-collection times by an order of magnitude or more (Humphry et al., 2012).

It is our hope that this technique will become the tool of choice for investigation of local symmetry and structure using electron diffraction, supplementing standard CBED techniques and finding a host of applications across many materials systems. The understanding gained of dynamical electron diffraction patterns (Gjønnes \& Moodie, 1965; Buxton et al., 1976; Goodman, 1975; Tanaka \& Tsuda, 2011; Morniroli et al., 2012) still applies to these new diffraction data sets, and the significant extra detail in D-LACBED patterns allows immediate and unambiguous determination of the presence of symmetry elements. Here, we have deliberately chosen a 'standard' transmission electron microscope without energy filtering or spectroscopy, and without even the smaller and more intense probe afforded by a field emission electron gun. There is no fundamental barrier to implementation of this technique on higher-performance machines; the sub-nm probe available on aberration-corrected machines should allow investigation of local symmetries close to the unit-cell level, although close control of beam shape, size and position will be necessary (Koch, 2011). Furthermore, the use of energy filtering, while unnecessary for the symmetry determination described here, produces more quantitative data. Looking forward, the increase in data quantity and quality produced by D-LACBED may also allow quantitative analysis of diffracted intensities to determine valence electron distributions (Zuo, 2004) to be performed on a wider range of materials, opening up the exciting possibility of examining strongly correlated systems (for example, high- $T_{\mathrm{c}}$ superconductors). Furthermore, we note that all other types of electron diffraction, such as SAED, CBED and even precession electron diffraction patterns, are a smaller sample of the more complete D-LACBED data set, and can be derived in a simple and straightforward manner from the 'digital' electron diffraction patterns shown here.
This work was funded by the EPSRC under grant No. EP/ J009229/1.

\section{References}

Buxton, B. F., Eades, J. A., Steeds, J. W. \& Rackham, G. M. (1976). Philos. Trans. R. Soc. London Ser. A, 281, 171-194.

Eades, J. (1980). Ultramicroscopy, 5, 71-74.

Friedel, G. (1913). C. R. Acad. Sci. Paris, 157, 1533-1536.

Gjønnes, J. \& Moodie, A. F. (1965). Acta Cryst. 19, 65-67.

Glazer, A. M. (1972). Acta Cryst. B28, 3384-3392.

Goodman, P. (1975). Acta Cryst. A31, 804-810.

Howard, C. J. \& Stokes, H. T. (1998). Acta Cryst. B54, 782-789.

Howard, C. J. \& Stokes, H. T. (2004). Acta Cryst. B60, 674-684.

Howard, C. J. \& Zhang, Z. (2004). Acta Cryst. B60, 249-251.

Humphry, M. J., Kraus, B., Hurst, A. C., Maiden, A. M. \& Rodenburg, J. M. (2012). Nat. Commun. 3, 730.

Jacob, D., Ji, G. \& Morniroli, J. P. (2012). Ultramicroscopy, 121, 61-71.

Johnson, A. W. S. (2007). Acta Cryst. B63, 511-520.

Kishida, K., Goto, K. \& Inui, H. (2009). Acta Cryst. B65, 405-415.

Koch, C. T. (2011). Ultramicroscopy, 111, 828-840.

Kossel, W. \& Möllenstedt, G. (1939). Ann. Phys. 428, 113-140.

Morniroli, J. P. \& Ji, G. (2009). Materials Research Society Symposium Proceedings, Vol. 1184, Electron Crystallography for Materials Research and Quantitative Characterization of Nanostructured Materials, edited by P. Moeck, S. Hovmoller, S. Nicolopoulos, S. Rouvimov, V. Petkov, M. Gateshki \& P. Fraundorf, pp. 37-48. Warrendale: Materials Research Society.

Morniroli, J. P., Ji, G. \& Jacob, D. (2012). Ultramicroscopy, 121, 42-60.

Politova, E. \& Venevtsev, Y. (1975). Mater. Res. Bull. 10, 319-325.

Reaney, I. M., Colla, E. L. \& Setter, N. (1994). Jpn. J. Appl. Phys. 33, 3984-3990.

Spence, J. C. H. (1993). Acta Cryst. A49, 231-260.

Stadelmann, P. (1987). Ultramicroscopy, 21, 131-145.

Steeds, J. W. \& Vincent, R. (1983). J. Appl. Cryst. 16, 317-324.

Tanaka, M., Saito, R., Ueno, K. \& Harada, Y. (1980). J. Electron Microsc. 29, 408-412.

Tanaka, M., Terauchi, M. \& Sekii, H. (1987). Ultramicroscopy, 21, 245-250.

Tanaka, M. \& Tsuda, K. (2011). J. Electron Microsc. 60, S245-S267. Terauchi, M. \& Tanaka, M. (1985). J. Electron Microsc. 34, 128-135.

Venevtsev, Y. N., Politova, E. D. \& Zhdanov, G. S. (1974). Ferroelectrics, 8, 489-490.

Vincent, R. \& Midgley, P. (1994). Ultramicroscopy, 53, 271-282.

Woodward, D. I. \& Reaney, I. M. (2005). Acta Cryst. B61, 387-399.

Zuo, J. M. (2004). Rep. Prog. Phys. 67, 2053-2103. 